\title{
Relationship of Time Interval between Antenatal Corticosteroid Administrations to Delivery with Respiratory Distress in Preterm Newborns
}

\author{
${ }^{1}$ Shyamala Guruvare, ${ }^{2}$ Barnali Basu, ${ }^{3}$ Lavanya Rai, ${ }^{4}$ Leslie Lewis, ${ }^{5}$ Shripad Hebbar, ${ }^{6}$ Prashanth Adiga
}

\begin{abstract}
Objectives: To assess the relationship of time interval between antenatal corticosteroid administration and delivery with respiratory distress in premature newborns.
\end{abstract}

Settings: Tertiary level teaching hospital

Population: Preterm deliveries between 28 and 34 weeks in the period of April 2011 to January 2013 where the mothers received one course of corticosteroid prophylaxis and fulfilled the selection criteria.

Materials and methods: Perusal of the hospital records was made to gather antenatal information and the details of delivery and the newborn. The patients were divided based on the number of doses of steroids received into three groups. The patients receiving complete steroid prophylaxis was further divided based on the time interval between steroid administration and delivery into five groups: 0 to 7 days, 8 to 14,15 to 21,22 to 28,29 days and beyond. We looked for association between neonatal respiratory outcomes and steroid-delivery intervals using Statistical Package for the Social Sciences version 16 (SPSS, Chicago, USA). Regression model was used to control for the confounding variables.

Results: There were 284 women who delivered preterm (up to 34 weeks of gestation) between April 2011 and January 2013 and fulfilled the selection criteria. The babies who received one (48) or no dose of steroids (14) had a higher incidence of respiratory distress than the ones who received a complete dose. This was statistically significant for babies born up to 32 weeks.

Among the rest 222 babies who received complete steroid prophylaxis, $138(62 \%)$ of the neonates born were admitted in $\mathrm{NICU}$, respiratory distress was seen in $62(28 \%)$. Eleven $(5 \%)$ of the babies required intubation and $22(9 \%)$ required surfactant therapy; there were $12(5 \%)$ neonatal deaths. Multivariable logistic regression analysis showed a slightly increased risk of respiratory morbidity with increased interval from administration to delivery (OR-0.87 for 8-14 days, 1.79 for $15-21$ days and 0.16 for $22-28$ days).

\footnotetext{
${ }^{1,6}$ Associate Professor, ${ }^{2}$ Resident, ${ }^{3,4}$ Professor

${ }^{5}$ Additional Professor

1-3,5,6 Department of Obstetrics and Gynecology, Kasturba Medical College, Manipal, Karnataka, India

${ }^{4}$ Department of Pediatrics, Kasturba Medical College, Manipal Karnataka, India

Corresponding Author: Shyamala Guruvare, Associate Professor, Department of Obstetrics and Gynecology Kasturba Medical College, Manipal, Karnataka, India, Phone: 08202545058, e-mail: shyamalarajgopal@hotmail.com
}

Conclusion: The risk of respiratory distress in preterm newborns increases beyond 2 weeks interval between antenatal corticosteroid administration and delivery.

Keywords: Antenatal steroids, Delivery interval, Respiratory distress.

How to cite this article: Guruvare S, Basu B, Rai L, Lewis L, Hebbar S, Adiga P. Relationship of Time Interval between Antenatal Corticosteroid Administrations to Delivery with Respiratory Distress in Preterm Newborns. Int J Infertil Fetal Med 2015;6(3): 128-132.

\section{Source of support: Nil}

Conflict of interest: None

Date of received: $27-10-15$

Date of acceptance: $12-11-15$

Date of publication: December 2015

\section{INTRODUCTION}

Prematurity with the associated complications such as respiratory morbidity, interventricular hemorrhage, necrotizing enterocolitis takes up the majority of NICU care. Antenatal corticosteroid administration is proven to reduce the incidence of these complications and is recommended at any threat of preterm delivery between 28 and 34 weeks of gestation.

The effect of the steroid administered is shown to be the maximum when delivery occurs between 24 hours and 7 days of the last dose of the regimen. ${ }^{1}$ When preterm delivery could be averted but the threat continued, researchers attempted administration of repeated doses (weekly) of steroids till term and observed conflicting results. While there are reports proving that multiple doses are safe and beneficial, ${ }^{2,3}$ some studies have raised concern over reduced birth weight and head circumference of those newborns exposed to repeated doses of steroids with no improvement in perinatal morbidity and mortality in comparison to placebo. ${ }^{4}$

Few studies focused to find the role if any of single complete regimen of steroid beyond 7 days of administration. ${ }^{5}$ We conducted this study with the objective of assessing the relationship of time interval between antenatal corticosteroid administration and delivery with neonatal respiratory distress. 


\section{MATERIALS AND METHODS}

A retrospective cohort study was conducted in the department of obstetrics and gynecology and neonatology of a tertiary care center.

Sample size calculation: Heljić et al (2009) $)^{6}$ conducted a study to determine the effects of antenatal corticosteroids and surfactant replacement on the severity and frequency of respiratory distress syndrome (RDS) in a cohort of premature infants born in Sarajevo, Bosnia and Herzegovina, from 2005 to 2007. The cohort consisted of 172 premature neonates with estimated gestational age between 26 and 34 weeks. Out of 172 neonates, 80 were treated antenatally with corticosteroids and 92/172 were not. Frequency of RDS was significantly lower in the corticosteroid group $(30 \%, 24 / 80)$ in relation to the control group (59\%, 54/92). Based on this information, we calculated sample size required for the study using the formula:

$$
\mathrm{n}=\left(\mathrm{Z}_{\alpha / 2}+\mathrm{Z}_{\beta}\right)^{2} \times\left[\mathrm{p}_{1}\left(1-\mathrm{p}_{1}\right)+\mathrm{p}_{2}\left(1-\mathrm{p}_{2}\right)\right] /\left(\mathrm{p}_{1}-\mathrm{p}_{2}\right)^{2}
$$

where $Z_{\alpha / 2}$ is the critical value of the Normal distribution at $\alpha / 2$ for a confidence level of $95 \%$ ( $\alpha$ is 0.05 and the critical value is 1.96), $Z_{\beta}$ is the critical value of the normal distribution at $\beta$ for a power of $80 \%, \beta$ is 0.2 and the critical value is 0.84 ) and $p_{1}$ and $p_{2}$ are the expected sample proportions of the two groups (30 and $58.7 \%$ or 0.3 and 0.59 respectively).

Accordingly minimum 43 patients were required to test the hypothesis and we considered all singleton preterm deliveries between 28 and 34 weeks in the period of April 2011 to January 2013. As it is not the institutional protocol to administer steroids to patients with preterm delivery at $<28$ weeks, patients who delivered at this gestational age were not included.

After obtaining permission from the Institutional Ethics Committee, perusal of the hospital records was made to gather information for the same.

For analysis, the newborns were divided into three groups:

- Group A: Newborns whose mothers received 2 doses of betamethasone or dexamethasone in pregnancy

- Group B: Newborns whose mothers received 1 dose of betamethasone or dexamethasone

- Group C: Newborns whose mothers received no steroid prophylaxis.

The group A (mothers who received a complete dose of steroid prophylaxis) was further subdivided into 3 groups based on the gestational age at which the steroids were administered:

- Patients receiving steroids between 28 and 30 weeks

- Patients receiving steroids between 30.1 and 32 weeks

- Patients receiving steroids between 32.1 and 34 weeks Only singleton pregnancies were chosen for the study.
The neonatal outcomes of these groups were then looked for. The adverse outcome measures included admission in the neonatal intensive care unit, respiratory distress, need for intubation and the need for surfactant therapy. Babies with congenital anomalies were excluded. In the absence of facility for amniotic fluid surfactant measurement, respiratory distress was diagnosed when any one of the following was present:

- Persistent tachypnea (Respiratory rate $>60 / \mathrm{min}$ )

- Presence of grunting and chest retractions.

- Persistent hypoxia (Inability to maintain saturation $>90 \%$ at room air)

- Requirement of assisted ventilation to maintain saturation and respiratory rate.

Chi-square test and multivariate logistic regression analysis were used to compare respiratory distress between babies depending on the number of steroid doses they received as well as to assess the relationship of time interval between antenatal corticosteroid administration and delivery with neonatal complications in premature babies receiving complete steroid prophylaxis.

\section{RESULTS}

Of the 438 preterm deliveries in the hospital during the study period, only 284 were eligible to be included (Flow Chart 1).

The patients were divided into three groups:

- Graph A: Newborns whose mothers received two doses of steroids antenatally-222

- Graph B: Newborns whose mothers received one dose-48

- Graph C: Newborns whose mothers received no steroid prophylaxis-14

The three groups of patients were comparable in terms of the general characteristics of age and parity. Seventyseven percent of these patients were delivered by cesarean delivery due to antenatal risk factors (Tables 1 and 2).

Flow Chart 1: Study design of the present study

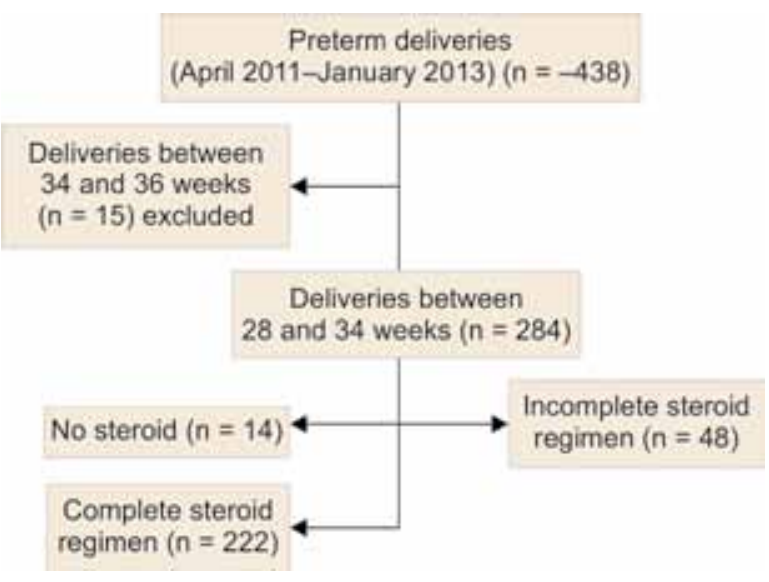


Table 1: Demographic characteristics of the patients

\begin{tabular}{llllll}
\hline Characteristic & & Group A 222 (\%) & Group B 48 (\%) & Group C 14 (\%) & ${ }^{*} p$-value \\
\hline Age (mean \pm standard deviation) & & $27 \pm 4.34$ & $25 \pm 3.6$ & $27 \pm 5.1$ & 0.76 \\
Parity & Primi & $146(76)$ & $37(77)$ & $8(57)$ & 0.22 \\
& Multi & $76(34)$ & $11(23)$ & $6(43)$ & - \\
Mode of delivery & Vaginal & $45(20)$ & $13(27)$ & $4(28)$ & 0.50 \\
& LSCS & $175(79)$ & $35(73)$ & $10(72)$ & - \\
\multirow{3}{*}{ Steroid } & Vacuum & $2(1)$ & 0 & 0 & - \\
& Betamethasone & $186(84)$ & $33(68)$ & NA & - \\
\hline
\end{tabular}

${ }^{*}$ Chi-square test, $p$-value $<0.05$ is significant

Table 2: Antenatal risk factors among the patients of the study

\begin{tabular}{lllll}
\hline Risk factor & Group $A(n=222)$ No (\%) & Group B $(n=48)$ No (\%) & Group C $(n=14)$ No (\%) & ${ }^{*} p$-value \\
\hline Pre-eclampsia & $70(31.5)$ & $29(60)$ & $6(42)$ & 0.001 \\
IUGR & $40(18)$ & $5(10)$ & 0 & 0.20 \\
Oligoamnios & $12(5.4)$ & $3(6)$ & 0 & 0.81 \\
GDM & $5(2.3)$ & 0 & $1(7)$ & 0.25 \\
Others & $30(13.5)$ & $5(10)$ & $3(21)$ & 0.56 \\
PPROM/ Preterm & $62(27.9)$ & $6(12)$ & $4(28)$ & 0.08 \\
None & $3(1.4)$ & 0 & 0 & \\
\hline
\end{tabular}

${ }^{*}$ Chi-square test, $p$-value $<0.05$ is significant

On account of prematurity, a large proportion of these babies 186 (65\%) were admitted in the neonatal intensive care unit. The babies at lesser gestational age were admitted for a more prolonged period of time than the ones at higher age. Among the neonatal outcomes studied the adverse outcome of respiratory distress (35\%) was most significantly present among babies of lesser gestational age. The other outcomes of interventricular hemorrhage, hyperbilirubinemia, neonatal seizures $(19,0.5$ and $0.5 \%)$ were present only in a small proportion among the three groups and hence could not be compared. The overall mortality in all the three groups was low (5\%).

Respiratory distress was significantly higher for babies at lower gestational age as well as in the babies who received incomplete or no dose of steroid (Table 3). We found however that even in babies with no or incomplete doses the incidence of respiratory distress was very low beyond 32 weeks of gestation.

For further analysis of the relationship between steroid delivery interval and respiratory distress in premature babies, only the babies who received complete steroid prophylaxis were considered. They were further divided into three groups depending on the gestational age at which steroids were given:

- Patients receiving steroids between 28 and 30 weeks-62

- Patients receiving steroids between 30.1 and 32 weeks-71

- Patients receiving steroids between 32.1 and 34 weeks-89

The babies in each group were then divided into groups based on the interval between the steroid administration and delivery and the incidence of respiratory distress compared among them. With increasing intervals and hence increasing gestational age at delivery, the incidence of respiratory distress was found to be decreasing (Graph 1).

Using multiple logistic regression analysis taking the interval of 0 to 7 days as the standard it was found that as the interval between steroid administration and delivery increased, the odds ratio for respiratory morbidity increased from 0.87 to 1.79 from interval of 2 to 3 weeks though it was not statistically significant. As this analysis was made after adjusting for the gestational age

Table 3: Comparison of respiratory distress with groups receiving complete, incomplete or no dose of steroid ${ }^{\dagger}$

\begin{tabular}{lllll}
\hline Delivery at gestational age & $\begin{array}{l}\text { Group A (2 doses) } \\
(n=222)\end{array}$ & $\begin{array}{l}\text { Group B (1 dose) } \\
(n=48)\end{array}$ & $\begin{array}{l}\text { Group C (no steroid) } \\
(n=14)\end{array}$ & ${ }^{*} p$-value \\
\hline 28 to 30 weeks $(n=91)$ & $29(13 \%)$ & $20(41 \%)$ & $6(42 \%)$ & 0.0004 \\
30 weeks 1d-32 weeks $(n=89)$ & $20(9 \%)$ & $9(18 \%)$ & $1(7 \%)$ & 0.03 \\
32 weeks 1d-34 weeks $(n=104)$ & $13(6 \%)$ & $4(8 \%)$ & 0 & 0.1 \\
\hline
\end{tabular}

${ }^{*}$ Test used: Chi-square test, $p$-value $<0.05$ is significant, ${ }^{\dagger}$ Percentages among patient groups 
at delivery, the effect of increasing gestational age with increasing interval of administration to delivery interval has been excluded (Table 4 and Graph 2). Beyond 3 weeks interval, the odds ratio again decreased as the pregnancies would be more than 32 weeks then.

\section{DISCUSSION}

Several studies and meta-analysis have proved beyond doubt the efficacy of antenatal corticosteroids in the prevention of complications in preterm neonates. Following the demonstration of their efficacy in 1972, steroids were introduced straight away; however questions still exist regarding the dosage, frequency and optimal timing of administration of antenatal steroids. ${ }^{1,5,7}$ The findings of our study also corroborate that babies who do not receive steroid prophylaxis are at increased risk for neonatal complications.

Unfortunately, it may be difficult to predict the exact time of delivery in mothers at risk of preterm delivery. Steroids are given for prophylaxis in the event that if the baby were to be delivered preterm it would have less of respiratory distress on account of acceleration of lung maturity. However in the event that preterm delivery was successfully averted and the baby still remains at risk for preterm delivery, would the steroid already be given beneficial? Is there a need for repeat dose of steroid if the interval exceeds 7 days and how many such repeat doses are needed?

Studies have suggested that steroids are most effective within 1 week of their administration. To be more precise, the maximum efficacy is within 2 days of administration requiring less of resuscitation and ventilation than the ones born at 2 to 7 days or $>7$ days interval. ${ }^{8}$

Similarly, Wilms et al in a group of 254 neonates observed an increased relative risk of respiratory morbidity

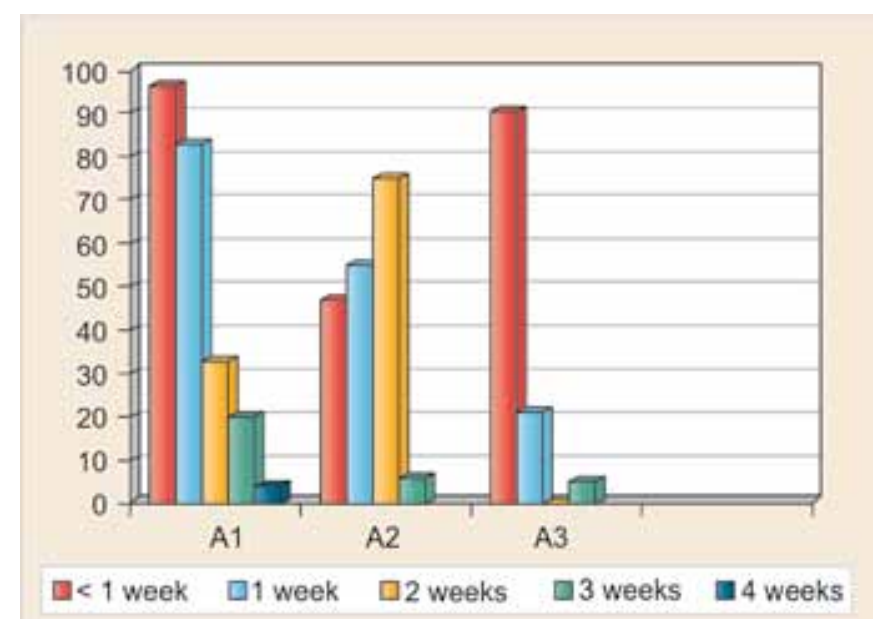

Graph 1: Incidence of respiratory distress with increasing interval from steroid administration to delivery in group $A(n=222)$
Table 4: Relationship between steroid delivery interval and neonatal respiratory outcome measures

\begin{tabular}{lll}
\hline \multicolumn{3}{c}{ Respiratory distress } \\
\hline Interval & Odds ratio & ${ }^{*} p$-value \\
\hline 8 to 14 days & $0.87(0.33-2.29)$ & 0.78 \\
15 to 21 days & $1.79(0.48-6.5)$ & 0.37 \\
22 to 28 days & $0.16(0.03-0.83)$ & 0.03 \\
$>29$ days & $0.01(0.001-0.03)$ & 0.99 \\
\hline
\end{tabular}

${ }^{*}$ Test used: Multiple logistic regression test after adjusting for gestational age at delivery, $p$-value $<0.05$ is significant

in babies delivered after 7 days of administration of steroids, particularly babies delivered at 28 to 30 weeks of gestation. ${ }^{9}$

Contrary to the above, our study demonstrates that the relative risk of neonatal respiratory distress increases only slightly with each passing week after antenatal corticosteroid administration irrespective of the gestational age at delivery in preterm babies; however the risk was the highest beyond the interval of 2 to 3 weeks.

Our groups predominantly included the newborns where pregnancies had to be terminated for various maternal complications and severe medical disorders. Though this would have contributed to the respiratory morbidity in the newborns, the groups were comparable in terms of demographic characteristics and the maternal risk factors, barring pre-eclampsia. Also it would have been more comprehensive had we considered other complications of prematurity such as intraventricular hemorrhage, necrotizing enterocolitis and leukomalacia in the analysis but their decreased incidence precluded that.

Similar to our observations, there are some other studies which demonstrate that the beneficial effect of antenatal steroids administered persist even beyond 1 week. They observed only an increased need for shortterm respiratory support, but not other measures of

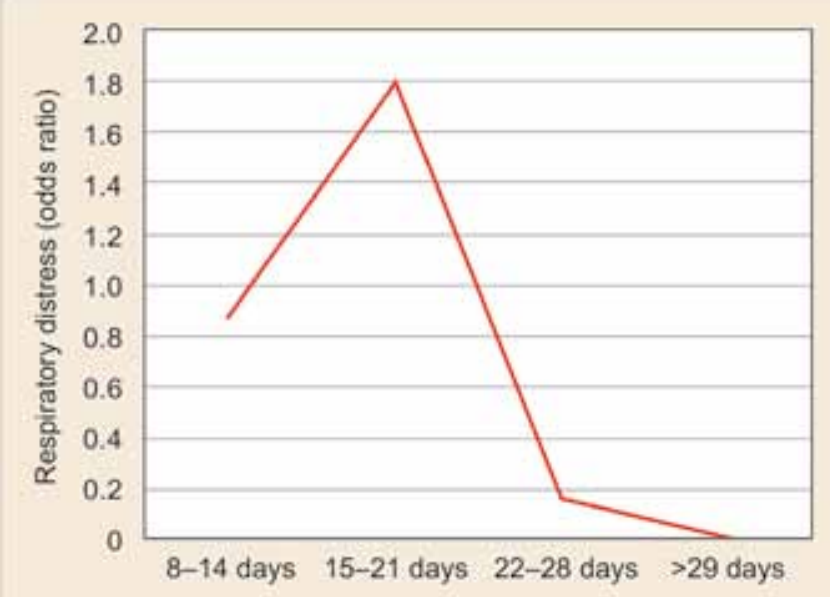

Graph 2: Risk of respiratory complications with increasing steroid to delivery interval in preterm babies 
neonatal morbidity when babies were born beyond 1 week after steroid administration. ${ }^{10,11}$

The findings of our study show that with increasing steroid-delivery interval, the risk of respiratory morbidity slightly increases beyond 2 to 3 weeks interval. Hence having given steroid prophylaxis once, a rescue dose may be required if the risk of preterm delivery persists and the interval crosses 2 weeks. Also it suggests further prospective research to explore the role of prophylactic antenatal steroid administration once in early third trimester in all high-risk pregnancies as well as the number of doses of steroid needed.

\section{CONCLUSION}

The risk of respiratory distress in preterm newborns increases beyond 2 weeks interval between antenatal corticosteroid administration and delivery.

\section{REFERENCES}

1. BrownfootFC, Gagliardi DI, Bain E, Middleton P,Crowther CA. Different corticosteroids and regimens for accelerating fetal lung maturation for women at risk of preterm birth. Cochrane Database of Systematic Reviews 2013, Issue 8.

2. McKinlay CJ, et al. Repeat antenatal glucocorticoids for women at risk of preterm birth: a cochrane systematic review. Am J Obstet Gynecol 2012;206(3):187-194.
3. Crowther CA, et al. Neonatal respiratory distress syndrome after repeat exposure to antenatal corticosteroids: a randomized controlled trial. Lancet 2006;367(9526):1913-1919.

4. Murphy KE, Hannah ME, Willan AR, Hewson SA, Ohlsson A, Kelly EN, Mathews SG, Saiqal S, Asztalos E, Ross S, et al. Multiple courses of antenatal corticosteroids for preterm birth (MACS): a randomized controlled trial. Lancet 2008 Dec 20;372(9656):2143-2151.

5. Wapner R, et al. Controversy: antenatal steroids. Clin Perinatol 2011;38(3):529-545.

6. Heljić S, Maksić H, Kalkan I, Krdalić B. The effects of antenatal corticosteroids and surfactant replacement on neonatal respiratory distress syndrome. Bosn J Basic Med Sci 2009;9(3):225-228.

7. Roberts D, Dalziel S. Antenatal corticosteroids for accelerating fetal lung maturation for women at risk of preterm birth. Cochrane Database Syst Rev 2006 Jul 19;3:CD004454.

8. Firouzabadi RD, Karbasi SA, Sekhavat, et al. Comparison of interval duration between single course antenatal corticosteroid administration and delivery on neonatal outcomes. J Turk Ger Gynecol Assoc 2011 Jun 1;12(2):86-89.

9. Wilms FF, et al. Relationship between the time interval from antenatal corticosteroid administration until preterm birth and the occurrence of respiratory morbidity. Am J Obstet Gynecol 2011;205(1):49.e1-7.

10. Vermillion ST, Bland ML, Soper DE. Effectiveness of a rescue dose of antenatal betamethasone after an initial single course. Am J Obstet Gynecol 2001 Nov;185(5):1086-1089.

11. Peaceman AM, Bajaj K, Kumar P, Grobmen WA. The interval between a single course of antenatal steroids and delivery and its association with neonatal outcomes. Am J Obstet Gynecol 2005 Sep-(3P62);193:1165-1169. 